\section{TECs support the SSC niche}

Testicular endothelial cells (TECs) are a key component of the spermatogonial stem cell (SSC) niche providing factors that are essential for SSC maintenance, according to new research published in Nature Communications. This discovery could aid fertility preservation for prepubertal boys who are diagnosed with cancer by enabling expansion and long-term culture of SSCs from testicular biopsy samples.

Expression of glial cell line-derived neurotrophic factor $(G d n f)$ mRNA in mouse-derived TECs after fibroblast growth factor 2 (FGF2) treatment was higher than in Sertoli cells. Numerous SSC colonies were observed after 3 weeks of co-culture with TECs, in contrast to SSC co-culture with Sertoli cells and peritubular myeloid cells. In vitro, bulsufan treatment decreased TEC proliferation and increased apoptosis, and testis tissue from bulsufan-treated

mice showed reduced TEC populations.

Transplantation of TECs into the testes of bulsufan-treated mice resulted in restoration of spermatogenesis. Furthermore, TEC injection into the testes immediately after bulsufan treatment protected spermatogenesis.

TEC co-culture with SSCs without exogenous GDNF was sufficient for 3D colony formation with typical SSC morphology; colonies were maintained in culture for $>3$ months and could be serially passaged. Transplantation of SSCs co-cultured with TECs into the testes of infertile mice restored fertility and resulted in the birth of live pups.

Comparison of the secretome of TECs with those of lung endothelial cells and liver endothelial cells showed that, as well as GDNF, insulin-like growth factor-binding protein 2, stromal cell-derived factor 1 and CXC motif chemokine 2 were

\section{Transplantation of TECs into the testes of} bulsufan-treated mice resulted in restoration of spermatogenesis

\section{INCONTINENCE}

upregulated in TECs. Addition of these factors along with FGF2 to feeder-cell-free cultures of mouse or human testicular cells was sufficient to maintain long-term SSC colony formation.

"For many years, scientists have been trying to reproducibly expand SSCs from testicular biopsies harvested from prepubertal boys newly diagnosed with cancer before gonadotoxic cancer treatment," Sandra Ryeom, corresponding author, tells Nature Reviews Urology. "Our work identifies TECs as a key population in the SSC niche and co-culture of SSCs with TECs enables consistently reproducible long-term expansion and maintenance of these stem cells," Ryeom continues. "Expansion of sperm stem cells will enable fertility preservation for patients who have no other options. This work is a key first step towards restoring fertility in boys and men who have survived childhood cancer."

Louise Stone

ORIGINAL ARTICLE Bhang, D. H. et al. Testicula endothelial cells are a critical population in the germline stem cell niche. Nat. Commun. 9, 4379 (2018)

\title{
eCoin device stops patients from spending a penny
}

An implantable neuromodulation device the size of a nickel, named 'eCoin', is safe and effective for treating urge urinary incontinence (UUI) associated with overactive bladder $(\mathrm{OAB})$, according to a paper in the Journal of Urology.

First-line therapy for $O A B$ is usually pharmacological but many patients do not respond, so alternatives are needed. In a prospective study carried out at seven institutes in the USA and New Zealand, the eCoin device was implanted in the medial lower leg of patients in an office-based procedure under local anaesthetic, after which efficacy and safety were assessed. "It was noteworthy how easy the eCoin device was to implant both for the patient and physician," comments corresponding author Scott MacDiarmid.
After the device was activated, electrical stimulation to the tibial nerve was delivered as a constant-current pulse (20 pulses per second; pulse width $0.2 \mathrm{~ms}$ ) with the stimulation pulse amplitude (0.5-15 mA) adjusted to the highest comfortable level for the patient, for 30-minute stimulation sessions every 2 days for 12 weeks.

Across the seven sites, 46 participants were implanted with the device,
Patients experienced... a median $71 \%$ relative reduction in number of episodes at 12 weeks 50 with a mean age of $63.4 \pm 11.5$ years; $98 \%$ of study participants were female. Patients experienced a median of 4.2 episodes of UUI per day at the baseline point (range 1-14.0). The median number of episodes of UUI dropped to 1.7 (range $0-10.7$ ) per day at 3 months and 1.5 (range $0-10.7$ ) episodes per day at 6 months, a median $71 \%$ relative reduction in number of episodes at 12 weeks $(P=0.001)$. Notably, after 6 months

of therapy, 31 of the 46 participants $(67.4 \%)$ continued to respond, with 22 (47.8\%) experiencing a reduction in UUI of $>75 \%$ and 11 (23.9\%) participants experiencing complete cessation of UUI. Patient-reported outcome measures also reflected these data, with an I-QOL change from baseline of nearly three times the minimally important difference for this measure, and $15 / 46$ (33\%) of patients feeling "very much better" according to the PGI-I questionnaire. No revision or removal was needed because of discomfort, pain, or infection at the implant or incision site.

MacDiarmid concludes: "We need to continue to offer therapies beyond medications that fail to reach the patient's treatment goal in the majority of cases. eCoin is an exciting new therapy that, with FDA approval, will provide us with a safe and effective treatment tool for these refractory patients."

Annette Fenner

ORIGINAL ARTICLE MacDiarmid, S. A. et al. Feasibility of a fully-implanted, nickel-sized and shaped tibial nerve stimulator for the treatment of overactive bladder syndrome with urgency urinary incontinence.J. Urol. https://doi.org/10.1016/j. juro.2018.10.017 (2018) 